(2) Open Access Full Text Article

\title{
Expression of SHP-I and SOCS6 in patients with acute leukemia and their clinical implication
}

\author{
This article was published in the following Dove Press journal: \\ OncoTargets and Therapy \\ 29 March 2017 \\ Number of times this article has been viewed
}

\author{
Jinmei Liu' \\ Yurong Zheng' \\ Ju Gao' \\ Guimei Zhu' \\ Kun $\mathrm{Gao}^{2}$ \\ Wenzhen Zhang ${ }^{3}$ \\ Fangyan $\mathrm{Shi}^{4}$ \\ Qing Zhang ${ }^{5}$ \\ 'Department of Hematology, Kailuan \\ General Hospital, ${ }^{2}$ Department of \\ Emergency, Tangshan Union Medical \\ College Hospital, ${ }^{3}$ Department of \\ Clinical Laboratory, Tangshan People's \\ Hospital, ${ }^{4}$ Department of Blood \\ Dialysis, Linxi Hospital, ${ }^{5}$ Department \\ of Ultrasonography, Tangshan, People's \\ Republic of China
}

\begin{abstract}
Background: To investigate the expression and clinical relevance of Src homology region 2 domain-containing phosphatase-1 (SHP-1) and suppressor of cytokine signaling 6 (SOCS6) in acute leukemia (AL).

Patients and methods: The enrolled AL patients were divided into three groups (newly diagnosed, relapsed, and complete remission [CR]). Healthy donors were also included as a control group in this study. Semi-quantitative reverse transcription polymerase chain reaction (RT-PCR) was performed to measure messenger RNA (mRNA) expression of SHP-1 and SOCS6. Statistical analysis was conducted to analyze the correlation between mRNA levels of SHP-1 and SOCS6 with patient outcomes.

Results: mRNA expression of SHP-1 was significantly lower in AL patients than that in healthy donors. The newly diagnosed or relapsed AL patients had lower mRNA levels of SHP-1 than the patients in CR. In contrast, SOCS6 mRNA expression was significantly higher in newly diagnosed or relapsed patients than that in patients in CR as well as healthy donors. However, mRNA levels of both SHP-1 and SOCS6 were positively correlated with the patient remission. The chemotherapyinduced remission rate was higher in patients with detectable SHP-1 or SOCS6 expression than in patients with undetectable SHP-1 or SOCS6 expression. Furthermore, the AL patients with detectable SHP-1 mRNA expression had lower incidence rate of invasive fungal infection.

Conclusion: The results suggest that expression patterns of SHP-1 and SOCS6 differ in AL patients. Despite the difference, expression of SHP-1 and SOCS6 is associated with favorable outcomes, suggesting an anticancer property of these two genes in AL.
\end{abstract}

Keywords: SHP-1, SOCS6, leukemia, prognosis, invasive fungal infection

\section{Introduction}

Leukemia is a group of heterogeneous and clonal hematopoietic malignancies, which is caused by mutations in hematopoietic stem cells or precursor cells. Although it can be found in any ages, leukemia often occurs in young adults with a higher rate of morbidity and mortality. ${ }^{1}$ The main cause of death includes severe infection, especially invasive fungal infection, bone marrow suppression, and so on. Therefore, early treatment of invasive fungal infection can increase the survival rate of patients with leukemia.

Undoubtedly, the development of cancer is closely linked with abnormal expression of oncogenes or tumor suppressor genes. ${ }^{2}$ Increased expression of oncogenes or silence of tumor suppressor genes is crucially involved in the tumorigenesis. Src homology region 2 domain-containing phosphatase-1 (SHP-1) is a tumor suppressor in lymphoma, leukemia, and other types of cancers. SHP-1, a member of the protein tyrosine phosphatase (PTP) family, plays an important role in suppressing the growthpromoting and oncogenic potentials of tyrosine kinases. ${ }^{3} \mathrm{SHP}-1$ is primarily expressed in hematopoietic cells and functions as a negative regulator of p-STAT3Tyr705 signal
Department of Hematology, Kailuan General Hospital, No 57, Xinhua East Road, Lubei District, Tangshan 063000 , People's Republic of China

Tel/fax +863I5302 7I52

Email drmei2015@sina.com

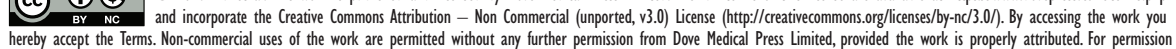
for commercial use of this work, please see paragraphs 4.2 and 5 of our Terms (https://www.dovepress.com/terms.php). 
transduction. It has been shown that the lower expression or silence of SHP-1 gene is associated with tumorigenesis.

SOCS6 is a member of suppressor of cytokine signaling (SOCS) family, which is a negative regulator of cytokine or growth factor-associated signaling. It is specifically involved in negative regulation of Janus kinase/signal transducer and activator of transcription (JAK/STAT) transduction pathway. ${ }^{4}$ It has been observed that SOCS6 is expressed in many types of tissues and that its expression is downregulated in several types of cancers.

Although it has been investigated in solid tumors, the expression of SHP-1 and SOCS6 has not been determined in hematological malignancies including leukemia. Especially, whether the expression levels of SHP-1 and SOCS6 affect the outcomes in patients with leukemia is unknown. In this study, messenger RNA (mRNA) expression of SHP-1 and SOCS6 in newly diagnosed or relapsed leukemia patients or patients in complete remission (CR) was investigated. The effect of expression levels of SHP-1 and SOCS6 on the incidence rate of chemotherapy-induced remission and invasive fungal infection was also determined.

\section{Patients and methods}

\section{Ethical statement}

Eligible patients provided written informed consent, in accordance with the Declaration of Helsinki. This study was approved by the ethics committee of Kailuan General Hospital.

\section{Patient characteristics}

A total of 62 patients ( 30 males and 32 females with a median age of 35 years [range: 14-70 years]) with acute leukemia (AL) including 36 newly diagnosed, 6 relapsed, and 20 in CR were enrolled in the study. Clinical data for these patients presenting to Tangshan Kailuan Hospital from August 2012 to October 2013 were evaluated. AL was diagnosed by using morphology, immunophenotype, cytogenetics, and molecular biology (MICM) of bone marrow cells. The patients were classified according to the World Health Organization (WHO) criteria published in 2008. As a control, 30 healthy donors (14 males and 16 females) with a median age of 34 years (range: 18-48 years) were enrolled in this study (Table 1).

\section{Patient treatment}

Newly diagnosed patients with acute myeloid leukemia (AML) received standard chemotherapy regimen of either idarubicin and cytarabine (IA) or daunorubicin plus cytarabine (DA). Patients with subtype M3 were treated with all-trans retinoic acid (ATRA) or ATRA plus arsenic
Table I Basic characteristics

\begin{tabular}{lllll}
\hline Characteristics & $\mathbf{n}$ & Gender & $\mathbf{n}$ & Age, years (range) \\
\hline Newly diagnosed & 36 & Male & 30 & \\
Relapsed & 6 & & & $35(14-70)$ \\
Complete remission & 20 & Female & 32 & \\
Healthy donors & 30 & Male & 14 & $34(18-48)$ \\
& & Female & 16 & \\
\hline
\end{tabular}

trioxide $\left(\mathrm{As}_{2} \mathrm{O}_{3}\right)$, or ATRA combining with low-dose DA or HA (cytarabine and homoharringtonine base). Patients with relapse or refractory $\mathrm{M} 3$ received $\mathrm{As}_{2} \mathrm{O}_{3}$ therapy. For ALL patients, chemotherapy regimen VDCLP (vincristine, daunorubicin, cyclophosphamide, L-asparaginase, prednisone) was administered. Patients with invasive fungal infection were treated with voriconazole or micafungin. ${ }^{5}$

\section{Reagents and equipment}

TRIzol $^{\circledR}$ Reagent was purchased from Thermo Fisher Scientific (Waltham, MA, USA). Moloney-Murine Leukemia Virus (M-MLV) reverse transcriptase was purchased from Promega Corporation (Fitchburg, WI, USA). Taq DNA polymerase was purchased from Sangon Biotech (Shanghai, China). DNA Thermal Cycler PE480 was purchased from PerkinElmer Inc. (Waltham, MA, USA). AlphaImager gel documentation system (AlphaImager 1200) was purchased from ProteinSimple (San Jose, CA, USA).

\section{Isolation of mononuclear cells}

Bone marrow samples $(4 \mathrm{~mL})$ from AL patients and healthy donors were freshly collected in a heparin tube at a concentration of $100 \mathrm{U} / \mathrm{mL}$ (heparin: bone marrow =1:4). Mononuclear cells were isolated by density gradient centrifugation using Ficoll-Paque. Cells were placed in an Eppendorf tube for the extraction of total RNA.

\section{Extraction of total RNA and semi- quantitative polymerase chain reaction (PCR)}

Total RNA was extracted by using TRIzol ${ }^{\circledR}$ Reagent according to the manufacturer's instructions. The quality of RNA was measured by the ratio of OD260/OD280. RNAs with ratio in the range of 1.6-2.0 were considered as high-quality RNA samples. The cDNA was synthesized by reverse transcription (RT) reaction. PCR was done by using DNA Thermal Cycler PE480 according to the manufacturer's instructions. The primers and PCR conditions were listed in Table 2. PCR products were separated on 2\% agarose gels and analyzed using an AlphaImager 1200. The relative SHP-1 expression level for each sample was calculated by absorbance value 
Table 2 PCR conditions and primers

\begin{tabular}{|c|c|c|c|}
\hline Gene & Primer sequences forward and reverse & PCR cycling conditions & Product length (bp) \\
\hline \multirow[t]{2}{*}{ SHP-I } & F: 5'-GGGCAGGCAGAGACGCT-3' & $94^{\circ} \mathrm{C} / 45^{\prime \prime} 56^{\circ} \mathrm{C} / 45^{\prime \prime}$ & 238 \\
\hline & R: 5'-CTTCTTGAAATGCTCCACCA-3' & $72^{\circ} \mathrm{C} / 60^{\prime \prime}$ & \\
\hline \multirow[t]{2}{*}{ socs6 } & F: 5'-TCTCACCATTGCTACTCCA-3' & $94^{\circ} \mathrm{C} / 45^{\prime \prime} 55^{\circ} \mathrm{C} / 45^{\prime \prime}$ & 466 \\
\hline & R: 5'-GAGTCCCTGATTGAATGCTC-3' & $72^{\circ} \mathrm{C} / 60^{\prime \prime}$ & \\
\hline \multirow[t]{2}{*}{$\beta$-Actin } & F: 5'-TCATCACCATTGGCAATGAG-3' & $94^{\circ} \mathrm{C} / 45^{\prime \prime} 55^{\circ} \mathrm{C} / 45^{\prime \prime}$ & 150 \\
\hline & R: 5'-CACTGTGTTGGCGTACAGGT-3' & $72^{\circ} \mathrm{C} / 60^{\prime \prime}$ & \\
\hline
\end{tabular}

Abbreviations: PCR, polymerase chain reaction; SHP-I, Src homology region 2 domain-containing phosphatase- I; SOCS6, suppressor of cytokine signaling 6; F, forward; $R$, reverse.

of SHP-1 mRNA divided by absorbance value of $\beta$-actin (internal control) mRNA.

\section{Statistical analysis}

All data were analyzed by using the statistical software SPSS13 (SPSS Inc., Chicago, IL, USA) and were represented as mean \pm standard deviation (SD). The non-parametric rank sum test was performed to determine the difference of SHP-1 and SOCS6 mRNA levels between the group of newly diagnosed patients or patients in CR and the group of healthy donors. The $\chi^{2}$ test was performed to determine the difference of the incidence rate of chemotherapy-induced remission and invasive fungal infection between groups of patients with detectable and undetectable SHP-1 or SOCS6 mRNA expression. $P<0.05$ was considered as statistically significant.

\section{Results}

\section{mRNA expression of SHP-I in patients with $A L$}

To explore the role of SHP-1 in AL, the mRNA expression of SHP-1 was first measured by PCR. To do this, the total
RNA from mononuclear cells of bone marrow from newly diagnosed $(n=36)$, relapsed $(n=6)$ AL patients, or patients in CR ( $\mathrm{n}=20)$ and also the total RNA from healthy donors $(n=30)$ were isolated. The RT was performed to synthesize cDNA for semi-quantitative PCR analysis. The expression level of SHP-1 for each sample was calculated by dividing the absorbance value of SHP-1 mRNA by the absorbance value of $\beta$-actin mRNA.

It was found that SHP-1 mRNA expression was detectable in all cases of healthy donors (detection rate: 100\%). In patients, the SHP-1 mRNA expression levels varied and the overall detection rate was lower than that in healthy donors. Of 36 newly diagnosed AL patients, 9 patients had detectable SHP-1 mRNA expression (detection rate: $25 \%$ ). It was observed that SHP-1 mRNA expression can be determined in 12 of 20 patients in $\mathrm{CR}$ with the detection rate being $60 \%$. Interestingly, none of 6 relapsed AL patients had detectable SHP-1 mRNA expression (Figure 1).

In addition to a higher detection rate, SHP-1 mRNA expression levels were also higher in healthy donors with a median expression level of 3.121 (range: $0.138-9.638, \mathrm{n}=30$ ).
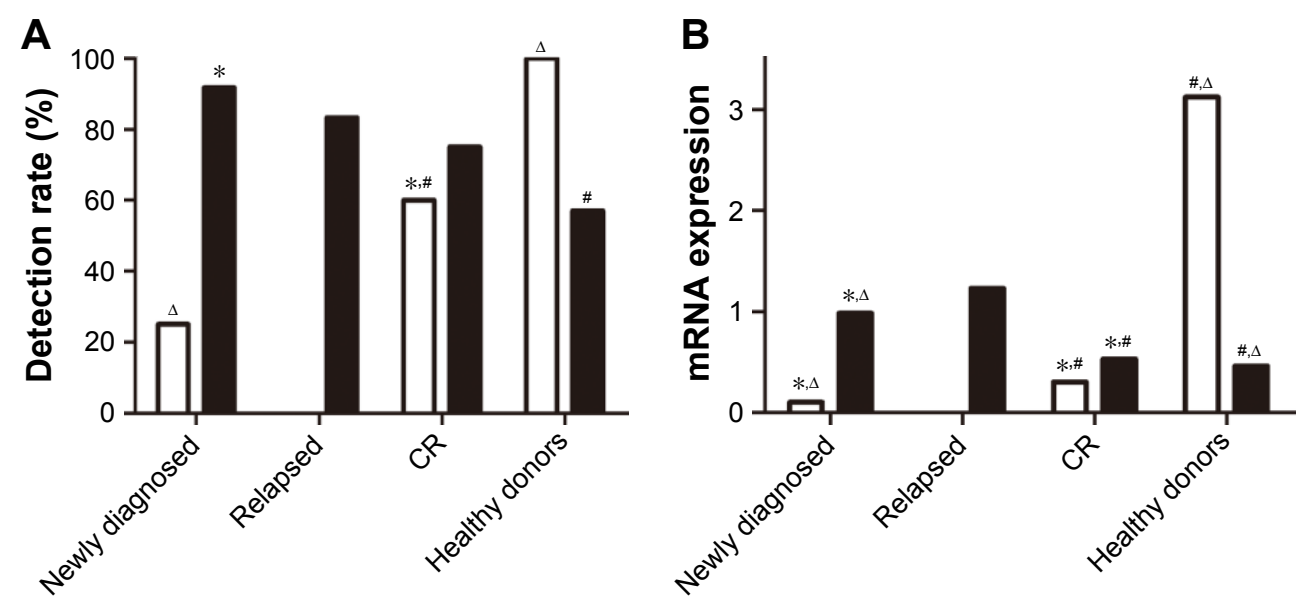

SHP-1 $\square$ SOCS6

Figure I mRNA expression of SHP-I and SOCS6 in AL patients. (A) mRNA expression of SHP-I in AL patients; (B) mRNA expression of SOCS6 in AL patients. Notes: *Versus healthy donor, $P<0.05$; " versus newly diagnosed, $P<0.05$; ${ }^{\circ}$ versus $C R, P<0.05$.

Abbreviations: AL, acute leukemia; CR, complete remission; mRNA, messenger RNA; SHP-I, Src homology region 2 domain-containing phosphatase-I; SOCS6, suppressor of cytokine signaling 6. 
In newly diagnosed AL patients, the median level of SHP-1 mRNA expression was 0.101 (range: $0-0.706, \mathrm{n}=36$ ), which is significantly lower than that in healthy donors $(P<0.05)$. In patients in CR, the median level of SHP-1 mRNA expression was 0.301 (range: $0-1.262, \mathrm{n}=20$ ), which is significantly higher than that in newly diagnosed patients, but still significantly lower than that in healthy donors (Figure 1).

\section{mRNA expression of SOCS6 in patients with $\mathrm{AL}$}

In contrast to SHP-1, the detection rate of SOCS6 mRNA expression was lower in healthy donors. Of 30 healthy donors, 17 donors had detectable SOCS6 mRNA expression (detection rate: $57 \%$ ). In patients, the overall detection rate of OCS6 mRNA expression was higher than that in healthy donors. Of the 36 newly diagnosed AL patients, 33 patients had detectable SOCS6 mRNA expression (detection rate: $91.7 \%$ ). It was observed that SOCS6 mRNA expression can be determined in 15 of 20 patients in $\mathrm{CR}$ with the detection rate being $75 \%$. In contrast to the finding that no relapsed AL patients had detectable SHP-1 mRNA expression, it was found that 5 of 6 relapsed AL patients had detectable SOCS6 mRNA expression (detection rate: $83.3 \%$, Figure 1).

Similar to a lower detection rate, SOCS6 mRNA expression levels were also lower in healthy donors with a median expression level of 0.458 (range: $0-2.866, n=30$ ). In newly diagnosed AL patients, the median level of SOCS6 mRNA expression was 0.982 (range: $0-9.598, \mathrm{n}=36$ ), which is significantly higher than that in healthy donors $(P<0.05)$. In patients in CR, the median level of SOCS6 mRNA expression was 0.529 (range: $0-2.555, \mathrm{n}=20$ ), which is significantly lower than that in newly diagnosed patients. Relapsed patients had the highest mRNA expression of SOCS6 with a median level of 1.228 (range: 0-9.6, $n=6$ ), which is significantly higher than that in newly diagnosed patients or patients in CR (Figure 1).

\section{Effect of SHP-I expression on patient outcomes}

Also, whether the expression levels of SHP-1 were associated with patient outcomes (rates of chemotherapy-induced remission and invasive fungal infection) need to be determined. To do this, the newly diagnosed or relapsed patients were divided into 2 groups based on detectable or undetectable SHP-1 mRNA expression. SHP-1 mRNA expression was detectable in 9 patients (all in newly diagnosed) and undetectable in 33 patients (27 in newly diagnosed and 6 relapsed). As shown in Table 3, chemotherapy-induced remission rate was higher in patients with detectable SHP-1 expression than the patients with undetectable SHP-1 expression. All the 9 patients were induced to remission with a remission rate being $100 \%$. Out of 33 patients with undetectable SHP-1 mRNA, 16 patients were induced to remission with a remission rate being $48 \%$, which is significantly lower than the patients with detectable SHP-1 mRNA expression.

Next, whether expression of SHP-1 impact invasive fungal infection in patients with newly diagnosed and relapsed AL was also determined. As shown in Table 3, the incidence rate of invasive fungal infection was significantly lower in patients with detectable SHP-1 mRNA expression than patients with undetectable SHP-1 mRNA expression. Of 9 patients with detectable SHP-1 expression, only 1 patient developed invasive fungal infection (incident rate: 11\%). In contrast, 32 of 33 patients with undetectable SHP-1 mRNA expression had invasive fungal infection (incident rate: $97 \%$ ).

\section{Effect of SOCS6 expression on patient outcomes}

The effect of expression levels of SOCS6 on the incident rates of chemotherapy-induced remission and invasive fungal infection in patients with AL was also determined. SOCS6 mRNA expression was detectable in 38 patients (33 in newly diagnosed and 5 in relapsed) and undetectable in 4 patients ( 3 in newly diagnosed and 1 in relapsed). Chemotherapyinduced remission rate was higher in patients with detectable SOCS6 mRNA expression than the patients with undetectable expression as shown in Table 3 . Of 38 patients with detectable SOCS6 mRNA expression, 25 patients were induced to remission (remission rate: 67\%). However, none of the 4 patients with undetectable SOCS6 mRNA expression was induced to remission (remission rate: $0 \%$ ), which

Table 3 Effect of SHP-I and SOCS6 mRNA expression on patient outcomes

\begin{tabular}{|c|c|c|c|c|c|c|c|c|}
\hline \multirow[t]{2}{*}{ Outcome } & \multicolumn{2}{|c|}{ SHP-I mRNA expression } & \multirow[t]{2}{*}{$\chi^{2}$} & \multirow[t]{2}{*}{$P$-value } & \multicolumn{2}{|c|}{ SOCS6 mRNA expression } & \multirow[t]{2}{*}{$\chi^{2}$} & \multirow[t]{2}{*}{$P$-value } \\
\hline & Detectable & Undetectable & & & Detectable & Undetectable & & \\
\hline Chemotherapy-induced remission & $100 \%(9 / 9)$ & $48 \%(16 / 33)$ & 5.79 & $<0.05$ & $66 \%(25 / 38)$ & $0 \%(0 / 4)$ & 4.06 & $<0.05$ \\
\hline Invasive fungal infection & II\% (I/9) & $97 \%(32 / 33)$ & 26.07 & $<0.01$ & $88 \%(29 / 38)$ & $100 \%(4 / 4)$ & 0.21 & $>0.05$ \\
\hline
\end{tabular}

Abbreviations: mRNA, messenger RNA; SHP-I, Src homology region 2 domain-containing phosphatase-I; SOCS6, suppressor of cytokine signaling 6. 
is significantly lower than patients with detectable SHP-1 mRNA expression.

In contrast to SHP-1, it was found that there was no significant difference of the incidence rate of invasive fungal infection between patients with detectable and undetectable SOCS6 mRNA expression. As shown in Table 3, the incidence rates of invasive fungal infection were $88 \%$ and $100 \%$ in patients with detectable and undetectable SOCS6 mRNA expression, respectively, without statistically significant difference. Of note, no statistical analysis was performed for patients in $\mathrm{CR}$ due to no invasive fungal infection observed in this group of patients.

\section{Discussion}

Protein tyrosine phosphorylation is considered to be a key regulatory mechanism in eukaryotic cell signal transduction, and it comprises two regulatory kinases protein-tyrosine kinases (PTKs) and PTPs. The dynamic balance between phosphorylation by PTKs and dephosphorylation by PTPs plays a pivotal role in the regulation of intracellular protein tyrosine phosphorylation levels, which is crucially involved in the control of cell growth, differentiation, and cell cycle. Disrupting the balance can result in abnormal cell proliferation and differentiation, leading to the development of various diseases. Elevated level of intracellular protein phosphorylation is one of the features in cancer cells. SHP-1 is one of the key phosphatases in signal transduction and negatively regulates intracellular protein phosphorylation. It has been shown that mRNA and protein levels of SHP-1 were decreased in tumor cells of leukemia and lymphoma as well as other neoplastic diseases. The methylation of SHP-1 gene promoter or transcription blockade of SHP-1 protein synthesis contributes to the decreased SHP-1 expression in several cancer cells. SHP-1 is usually expressed in normal cells, but its expression is reduced in AL and increased in patients in CR. ${ }^{2}$ It was reported that SHP-1 plays a role in CML disease progression. Reduced expression of SHP-1 might free BCR-ABL1 to recruit and activate JAK2. ${ }^{6}$ It was found that newly diagnosed AL patients had reduced expression of SHP-1 mRNA compared with healthy donors. When a $\mathrm{CR}$ is reached, these patients have elevated expression of SHP-1 compared with newly diagnosed patients. Importantly, it was observed that SHP-1 expression is positively correlated with the disease remission in leukemia, confirming the role of SHP-1 as a tumor suppressor gene during the pathogenesis of leukemia.

Proliferation and differentiation of hematopoietic cells are regulated by many soluble peptides through JAK-STAT pathway. JAK-STAT pathway plays an important role in the development of cancers and autoimmune diseases. ${ }^{7}$ Heuser et $\mathrm{al}^{8}$ showed that STAT signaling is involved in self-renewal of leukemia stem cells. Normally, there are also other regulators, such as SOCS family, to deactivate JAK-STAT signaling. SOCS6 exerts its function through JAK-STAT pathway. ${ }^{9}$ The SOCS family consists of eight proteins in humans, termed as SOCS1-7 and CISH. SOCS1-3 and CISH primarily regulate cytokine receptor, whereas SOCS4-7 are major regulators of tyrosine kinase receptor. These proteins play important roles not only in the development and homeostasis of body but also in the inhibition of tumor growth and anti-inflammation..$^{10}$ It has been shown that SOCS6 is a stem cell factor-dependent negative feedback regulator and possess targeting specificity of SOCS family bearing ubiquitin ligase. ${ }^{11}$ This study found that newly diagnosed AL patients had higher mRNA expression level of SOCS6 compared with healthy donors. mRNA expression level of SOCS6 is decreased in patients in CR. Furthermore, it was observed that SOCS6 expression was positively correlated with chemotherapy-induced remission in AL patients, which is consistent with a previous study. ${ }^{12}$

In summary, it was found that SHP-1 mRNA expression is lower in patients with newly diagnosed or relapsed leukemia and is higher in patients with remission. In contrast, it was found that SOCS6 expression is higher in patients with newly diagnosed or relapsed leukemia and is lower in patients with remission. However, expression levels of both SHP-1 and SOCS6 mRNA were positively correlated with remission as chemotherapy-induced remission rate was higher in patients with detectable SHP-1 or SOCS6 expression than patients with undetectable SHP-1 or SOCS6 expression. These results suggest that different mechanisms might be involved for SHP-1 and SOCS6 as tumor suppressor genes. Gene therapy has become a promising treatment for leukemia in recent years. Progress has been made in utilizing the suppression of JAK-STAT signal transduction to treat leukemia patients. ${ }^{13}$ Currently, investigation on association between genetic factors and susceptibility to fungal infection is in progress. ${ }^{14}$ Therefore, early prediction of susceptible genes can be clinically important in donor selection of bone marrow transplantation and in individualized medicine for the treatment of leukemia.

\section{Acknowledgment}

This work was supported by the grant from the Hebei Province Health Department of Scientific Research Fund Project (Funding No 20150837). 


\section{Disclosure}

The authors report no conflicts of interest in this work.

\section{References}

1. Ferrara F, Schiffer CA. Acute myeloid leukaemia in adults. Lancet. 2013;381:484-495.

2. Meng Z, Luo JM. Effect of methylation inhibitor in the treatment of leukemia. Zhongguo Shi Yan Xue Ye Xue Za Zhi. 2013;21:536-538.

3. Wu C, Sun M, Liu L, Zhou GW. The function of the protein tyrosine phosphatase SHP-1 in cancer. Gene. 2003;306:1-12.

4. Kabir NN, Sun J, Rönnstrand L, Kazi JU. SOCS6 is a selective suppressor of receptor tyrosine kinase signaling. Tumour Biol. 2014;35:10581-10589.

5. Yeh TC, Liu HC, Hou JY, et al. Severe infections in children with acute leukemia undergoing intensive chemotherapy can successfully be prevented by ciprofloxacin, voriconazole, or micafungin prophylaxis. Cancer. 2014;120:1255-1262.

6. Kaeda J, Neuman D, Bonecker S, et al. Differential expression of SHP-1 in chronic myeloid leukemia. Leuk Lymphoma. 2015;56(5):1547-1549.

7. Laurence A, Pesu M, Silvennoinen O, O'Shea J. JAK kinases in health and disease: an update. Open Rheumatol J. 2012;6:232-244.
8. Heuser M, Sly LM, Argiropoulos B, et al. Modeling the functional heterogeneity of leukemia stem cells: role of STAT5 in leukemia stem cell self-renewal. Blood. 2009;114:3983-3993.

9. Gupta S, Mishra K, Surolia A, Banerjee K. Suppressor of cytokine signalling-6 promotes neurite outgrowth via JAK2/STAT5-mediated signalling pathway, involving negative feedback inhibition. PLoS One. 2011;6:e26674.

10. Trengove MC, Ward AC. SOCS proteins in development and disease. Am J Clin Exp Immunol. 2013;2:1-29.

11. Zadjali F, Pike AC, Vesterlund M, et al. Structural basis for c-KIT inhibition by the suppressor of cytokine signaling 6 (SOCS6) ubiquitin ligase. J Biol Chem. 2011;286:480-490.

12. Sasi W, Jiang WG, Sharma A, Mokbel K. Higher expression levels of SOCS 1,3,4,7 are associated with earlier tumour stage and better clinical outcome in human breast cancer. BMC Cancer. 2010;10:178.

13. Jung JH, won TR, Jeong SJ, et al. Apoptosis induced by tanshinone IIA and cryptotanshinone is mediated by distinct JAK/STAT $3 / 5$ and SHP $1 / 2$ signaling in chronic myeloid leukemia K562 cells. Evid Based Complement Alternat Med. 2013;2013:805639.

14. Lionakis MS. Genetic susceptibility to fungal infections in human. Curr Fungal Infect Rep. 2012;6:11-22.
OncoTargets and Therapy

\section{Publish your work in this journal}

OncoTargets and Therapy is an international, peer-reviewed, open access journal focusing on the pathological basis of all cancers, potential targets for therapy and treatment protocols employed to improve the management of cancer patients. The journal also focuses on the impact of management programs and new therapeutic agents and protocols on

\section{Dovepress}

patient perspectives such as quality of life, adherence and satisfaction. The manuscript management system is completely online and includes a very quick and fair peer-review system, which is all easy to use. Visit http://www.dovepress.com/testimonials.php to read real quotes from published authors. 